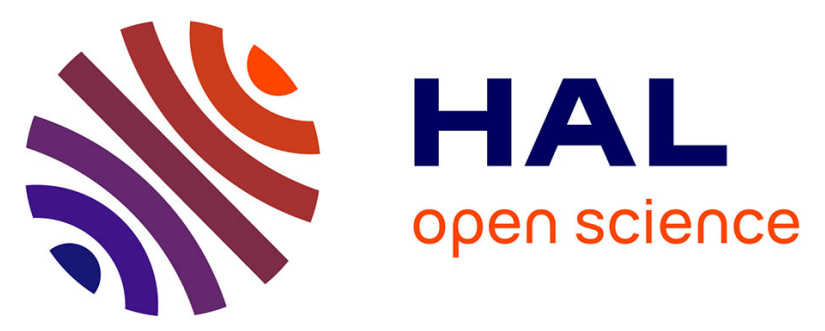

\title{
Effect of tamoxifen and fulvestrant long-term treatments on ROS production and (pro/anti)-oxidant enzymes mRNA levels in a MCF-7-derived breast cancer cell line
}

Eric Badia, Marion Morena, Céline Lauret, Abdelhay Boulahtouf, Nathalie Boulle, Vincent Cavaillès, Patrick Balaguer, Jean-Paul Cristol

\section{To cite this version:}

Eric Badia, Marion Morena, Céline Lauret, Abdelhay Boulahtouf, Nathalie Boulle, et al.. Effect of tamoxifen and fulvestrant long-term treatments on ROS production and (pro/anti)-oxidant enzymes mRNA levels in a MCF-7-derived breast cancer cell line. Breast Cancer, 2016, 23 (5), pp.692 - 700. 10.1007/s12282-015-0626-7 . hal-01802789

\section{HAL Id: hal-01802789 \\ https://hal.umontpellier.fr/hal-01802789}

Submitted on 25 Nov 2019

HAL is a multi-disciplinary open access archive for the deposit and dissemination of scientific research documents, whether they are published or not. The documents may come from teaching and research institutions in France or abroad, or from public or private research centers.
L'archive ouverte pluridisciplinaire HAL, est destinée au dépôt et à la diffusion de documents scientifiques de niveau recherche, publiés ou non, émanant des établissements d'enseignement et de recherche français ou étrangers, des laboratoires publics ou privés. 


\title{
Effect of tamoxifen and fulvestrant long-term treatments on ROS production and (pro/anti)-oxidant enzymes mRNA levels in a MCF-7-derived breast cancer cell line
}

\author{
Eric Badia1,7 • Marion Morena1,7 • Celine Lauret1,7 • Abdelhay Boulahtouf2,3,4 Nathalie \\ Boulle5,6 Vincent Cavaille`s2,3,4 • Patrick Balaguer2,3,4 • Jean Paul Cristol1
}

Eric Badia : e-mail: eric.badia@univ-montp1.fr

1 PhyMedExp, UMR CNRS 9214, Inserm U1046, Université de Montpellier, 34295

Montpellier Cedex 5, France

2 IRCM, Institut de Recherche en Cancérologie de Montpellier, 34298 Montpellier, France

3 INSERM, U1194, 34298 Montpellier, France

4 ICM, Institut régional du Cancer de Montpellier, 34298 Montpellier, France

5 CHU de Montpellier Arnaud de Villeneuve, 371, Avenue Doyen Gaston Giraud, 34295

Montpellier Cedex 5, France

6 Université de Montpellier, 34090 Montpellier, France

7 INSERM, U1046, IURC, 641 Avenue du Doyen Gaston Giraud, 34093 Montpellier, France

Keywords Cancer_Breast_MCF-7_Anti-estrogens _Oxidative stress

\begin{abstract}
Background Reactive oxygen species (ROS) are key players in the apoptotic effects induced by short-term tamoxifen treatment of breast cancer cells, but also in acquired resistance following long-term treatment. Whereas the use of the selective estrogen receptor downregulator fulvestrant is promising, especially in patients who develop tamoxifen resistance, only few studies addressed its implication in the modulation of cellular redox status.

Methods The regulation of (pro/anti)-oxidant players were first investigated at the mRNA level in a MCF-7- derived cell line after short-term ( $24 \mathrm{~h})$ estradiol treatment. Long-term antiestrogen treated MCF-7 derived cell lines were also developed: 3 months of 4 hydroxytamoxifen alone (MCF7L-OHTLT) or followed by 3 months of fulvestrant (MCF7LICILT). Growth properties, hormone sensitivity, receptor content, ROS production and relative mRNA expression of pro or antioxidant enzymes were evaluated in these long-term treated cell lines.

Results Short-term estradiol treatment showed a hormone sensitivity of Nox2, GPx1, GPx2 and SOD1 mRNA levels. The long-term fulvestrant treatment (3 months) of MCF7LOHTLT led to a reduced level of ROS production accompanied with a drastic drop of the accessory protein p22phox mRNA. This ROS reduction, although not clearly related to antioxidant enzymes level, seems to be involved in fulvestrant sensitivity of long-term anti-estrogen treated cells, as suggested by the effects of antiradical tempol treatment.

Conclusion When compared to long-term 4-hydroxytamoxifen- treated breast cancer cells, addition of fulvestrant treatment was able to diminish ROS production and p22phox mRNA level, and made cells more sensitive to growth inhibition induced by tempol. These effects may be a valuable asset of the fulvestrant treatment.
\end{abstract}




\section{Introduction}

Beside non-specific damages to DNA, lipids or proteins, it has long been established that, depending of their level of production, reactive oxygen species could also influence many cellular processes involved in cancer development such as proliferation, apoptosis and senescence [1-5]. Reactive oxygen species (ROS) that are derived from the metabolism of molecular oxygen include singlet oxygen (1O2), superoxide anion radical (O2 -.), hydrogen peroxide (H2O2), and hydroxyl radical (HO.). Moderate ROS formation is often required for signaling by growth factors [6] or for triggering important pathways such as cell migration and invasion, which are the initial steps of metastasis [7]. A critical balance between ROS generation and antioxidant defense systems is essential to limit deleterious effects of these ROS and involves proteins, such as catalase (CAT), superoxide dismutases (SOD), glutathione peroxidases (GPx) [5, 8, 9].

The NADPH oxidase (Nox) family is composed of 7 catalytic subunits termed Nox $1-5$ and Duox1-2. These Nox enzymes are transmembrane proteins that transport electrons to reduce oxygen to superoxide. They are sources of ROS and are currently considered for their role in cancer cells through various processes including cell growth, apoptosis, angiogenesis, invasion and metastasis [3]. Nox1-4 form a complex with both the small membrane-bound p22phox protein and some other regulatory cytosolic proteins, in order to facilitate the electron transfer, whereas Nox5 binds calcium ions [10].

Estrogens have proven to alter the subtle equilibrium between generation and suppression of ROS in vessels [11], uterus [12], and also in breast cancer cells [13]. Some of these effects might participate to the protective effect of estradiol on cardiovascular system in females. Breast cancer adjuvant therapies could also involve generation of ROS that could interfere with their mechanism of action. The anti-estrogen tamoxifen is the drug most often used for long-term (LT) treatment of early breast cancer. It was established that tamoxifen induces apoptosis in many cells, and this phenomenon could be mediated by ROS generation in the mitochondrion of breast cancer cells [14]. A serious limitation of tamoxifen endocrine therapy is the inevitable appearance of resistance that concerns approximately $50 \%$ of patients [15].

Several mechanisms underlying this phenomenon have been described [16, 17]. Interestingly, Cho et al. established that tamoxifen resistance could be reversed by silencing mitochondrial manganese SOD [18], indicating that ROS could be important players in the emergence of resistant cells. By contrast, only few studies concerned the effects of ROS on resistance to the pure anti-estrogen fulvestrant (ICI182,780), although it confers clinical benefits in a subset of patients refractory to tamoxifen therapy [19-21]. As a redox imbalance could be a key factor involved in cancer cell survival [22], the effect of long-term anti-estrogen treatment on redox equilibrium remains an important question. Being currently involved in several aspects of long-term anti-estrogen treatments [19, 23, 24], we were interested in comparing ROS production and mRNA expression of pro and antioxidant enzymes, in MCF-7 breast cancer cells with long-term treatments with hydroxytamoxifen (OHT) followed or not by fulvestrant.

\section{Materials and methods}

\section{Chemicals and materials}

Culture medium and fetal calf serum (FCS) were obtained from Life Technologies Inc. (Cergy-Pontoise, France). 17b-estradiol (E2), Thiazolyl Blue Tetrazolium bromide (MTT), 
DCFH-DA, lucigenin and tempol were from Sigma-Aldrich Inc. (St Louis, MO, USA). 4Hydroxytamoxifen and fulvestrant were a kind gift from Zeneca (Macclesfield, UK). RNA extraction kit was from Qiagen (Courtaboeuf, France). Superscript II reverse transcriptase was from Invitrogen (Cergy-Pontoise, France). The polyclonal antibodies against ERa (HC20) and Actin were, respectively, from Santa Cruz Biotechnology (California, USA) and Sigma Aldrich (St Quentin, France). The antirabbit secondary antibody was from Jackson ImmunoResearch (Suffolk, UK). The ECL detection system was from Thermo Fisher Scientific (Illkirch, France) and the Hoechst reagent from Sigma Aldrich (St Quentin, France). For quantitative PCR, SYBR Green Master mix and Light Cycler Real-Time PCR system were from Roche Applied Science (Meylan, France). For luminescence analysis, the Centro LB 960 luminometer was from Berthold (Thoiry, France).

\section{Cell lines and culture conditions}

MCF-7 is a cell line that was first isolated in 1970 from the breast tissue of a 69-year-old Caucasian woman. MCF7L cells (derived from MCF-7 cell line by stable transfection of a luciferase gene under constitutive promoter) were routinely maintained in DMEM-F12 medium supplemented with $5 \%$ FCS (FCS medium). During long term treatments, MCF7L cells were grown either in: DMEM-F12 (phenol red-free) supplemented with $3 \%$ dextrancoated charcoal-treated FCS (DCC) to obtain MCF7L-DCCLT, or with DCC medium with 10nM of 4-hydroxytamoxifen to obtain MCF7L-OHTLT, or with DCC medium with 10nM ICI182,780 to obtain MCF7LICILT cell line (vehicle was ethanol). Before mRNA analysis, cells were hormone-deprived in DCC medium for 4 days.

\section{Cell growth analysis}

Cell growth analysis was tested by using the MTT assay. After 4 days of hormone deprivation, cells were seeded at a density of about 1000 cells/well in 96-well tissue culture plates in DCC medium. The next day (day 0), $10 \mathrm{nM}$ E2 or $10 \mathrm{nM}$ OHTam or $10 \mathrm{nM}$ ICI182,780 or vehicle alone (ethanol) were added to the cultures ( $\mathrm{n}=6$ wells/each condition). Medium was changed every 2 days. At day $8,0.5 \mathrm{mg} / \mathrm{mL}$ of MTT was added to selected wells at $37^{\circ} \mathrm{C}$ for $4 \mathrm{~h}$. MTT solution was then removed and the plates were air-dried. Formazan crystals were then solubilized in DMSO. Absorbance was read at $540 \mathrm{~nm}$ on a spectrophotometer using a reference filter of $620 \mathrm{~nm}$ and results were normalized to the cell density at day 0 . RNA extraction and quantitative PCR analysis Total RNA was extracted from cells using a Qiagen kit, then, $1 \mathrm{lg}$ of total RNA was reverse transcribed using the Superscript II reverse transcriptase. Real-time quantitative Polymerase Chain Reaction (qPCR) was performed on a Light Cycler Real-Time PCR system. Each reaction included $2 \mathrm{lL}$ of SYBR Green Master mix, 0.5 lL of each primer dilution (10 lM), $2.5 \mathrm{lL}$ of RT product (1:10 dilution) and $\mathrm{H} 2 \mathrm{O}$ to $10 \mathrm{lL}$. For each sample, results were normalized to the $18 \mathrm{~S}$ mRNA levels (reference gene). Primers corresponding to human sequences used to assess gene expression were as follows:

18S forward:

18S reverse:

Nox2 forward:

Nox2 reverse:

Nox5 forward:

Nox 5 reverse:

p22phox

forward:
AACGAGACTCTGGCATGCTAACTA, CGCCACTTGTCCCTCTAAGAA; GCCCAAAGGTGTCCAAGCT, TCCCCAACGATGCGGATAT; CTGAACATCCCCACCATTGCTC, GACCGAATGTGCAGCCAGATAG;

CGCTTCACCCAGTGGTACTTTG, 
p22phox

reverse:

CAT forward:

CAT reverse:

GPx1 forward:

GPx1 reverse:

GPx2 forward:

GPx2 reverse:

SOD1

forward:

SOD1 reverse:

Nrf2 forward:

Nrf2 reverse:

Keap1

forward:

Keap1 reverse:

NQO1

forward:

NQO1

reverse:
ACGGCGGTCATGTACTTCTG;

TGTTGAAGATGCGGCGAGAC, GGGTACTTTCCTGTGGCAATGG;

GACCATTGACATCGAGCCTGAC, TAGATGAAAACCCCCCCGAGAC;

TGAATGGGCAGAACGAGCATC, CGGTCATGAGGGAAAATGGGTC;

AAAGATGGTGTGGCCGATGTG, ACCTTTGCCCAAGTCATCTGC; ACACGGTCCACAGCTCATC, TGTCAATCAAATCCATGTCCTG;

ATTGGCTGTGTGGAGTTGC, CAGGTTGAAGAACTCCTCTTGC;

ATGTATGACAAAGGACCCTTCC, TCCCTTGCAGAGAGTACATGG.

\section{Measurement of basal intracellular ROS by DCFHDA method}

In the same 96-well plate, cells were seeded at different densities (from 5000 to 50,000 cells per well). $24 \mathrm{~h}$ later, basal intracellular ROS production was detected as follows. Medium was removed and cells were incubated with $200 \mathrm{lL}$ of $20 \mathrm{lMDCFH}-\mathrm{DA}$ during $15 \mathrm{~min}$. Wells were then rinsed and filled with $200 \mathrm{lL}$ medium without serum. The fluorescence of 2,7dichlorofluorescein was recorded using a Berthold Tristar 2 LB942 spectrofluorometer during $2 \mathrm{~h}$. Fluorescence was normalized by protein determination (lowry) of cells in wells.

Measurement of membrane production of superoxide anion by lucigenin-derived chemiluminescence

Superoxide production was measured in total cell extract by using lucigenin-derived chemiluminescence as described in [25]. Cells were collected, trypsinized, and washed three times in PBS. They were then submitted to two successive freeze-thaw cycles in buffer A $(0.1$ $\%$ Tween 20, $25 \mathrm{mM}$ Tris $\mathrm{pH} 7.4$ ) with 15 s sonication before doing the later cycle. Aliquots of extracts were kept frozen at-80_C. Total protein determination was performed by using the Lowry method. Chemiluminescence was recorded during 45 min on a mixture containing: $0.66 \mathrm{lg} / \mathrm{lL}$ of protein, $0.55 \mathrm{mM}$ of NADPH, and $7.4910-5$ Mof lucigenin in buffer B $(1 \mathrm{mM}$ $\mathrm{MgCl} 2,100 \mathrm{lM} \mathrm{CaCl} 2,25 \mathrm{mM}$ Tris $\mathrm{pH} 7.4)$. DPI inhibitor $(0.1 \mathrm{mM})$ was added in controls. ROS measurement of each long-term treated cell line was achieved in parallel and in the same assay with that of MCF7L control cell line.

\section{Statistical analysis}

Data are shown as means and SEM. Statistical analysis was performed using GraphPad Prism software (San Diego, California). For Figs. 2, 3, 5, 6, a one-way ANOVA followed by a post hoc Bonferroni test was used to compare values. For Fig. 4, Student's t test was used to 
compare values of two groups of data (with or without E2 treatment). In figures, bars with different index letters are significantly different, with a threshold for statistical significance set at $\mathrm{p} \backslash 0.05$.

\section{Results}

\section{Effect of estradiol on pro or antioxidant enzymes mRNA expression in MCF-7 cells}

The mRNA expressions of NADPH oxidase homologues and accessory proteins have been previously analyzed by Juhasz et al. by using real-time PCR in a number of human tumor cell lines [26]. In this study, it was found that breast cancer-derived MCF-7 cell line contained moderate levels of Nox1, Nox 2 (the more abundant) and Nox 5 mRNA (levels of Nox3, Nox4 or Duox1-2 mRNA were negligible). Moreover, MCF-7 cell line was among those that contained the highest level of the accessory protein $\mathrm{p} 22^{\text {phox }}$
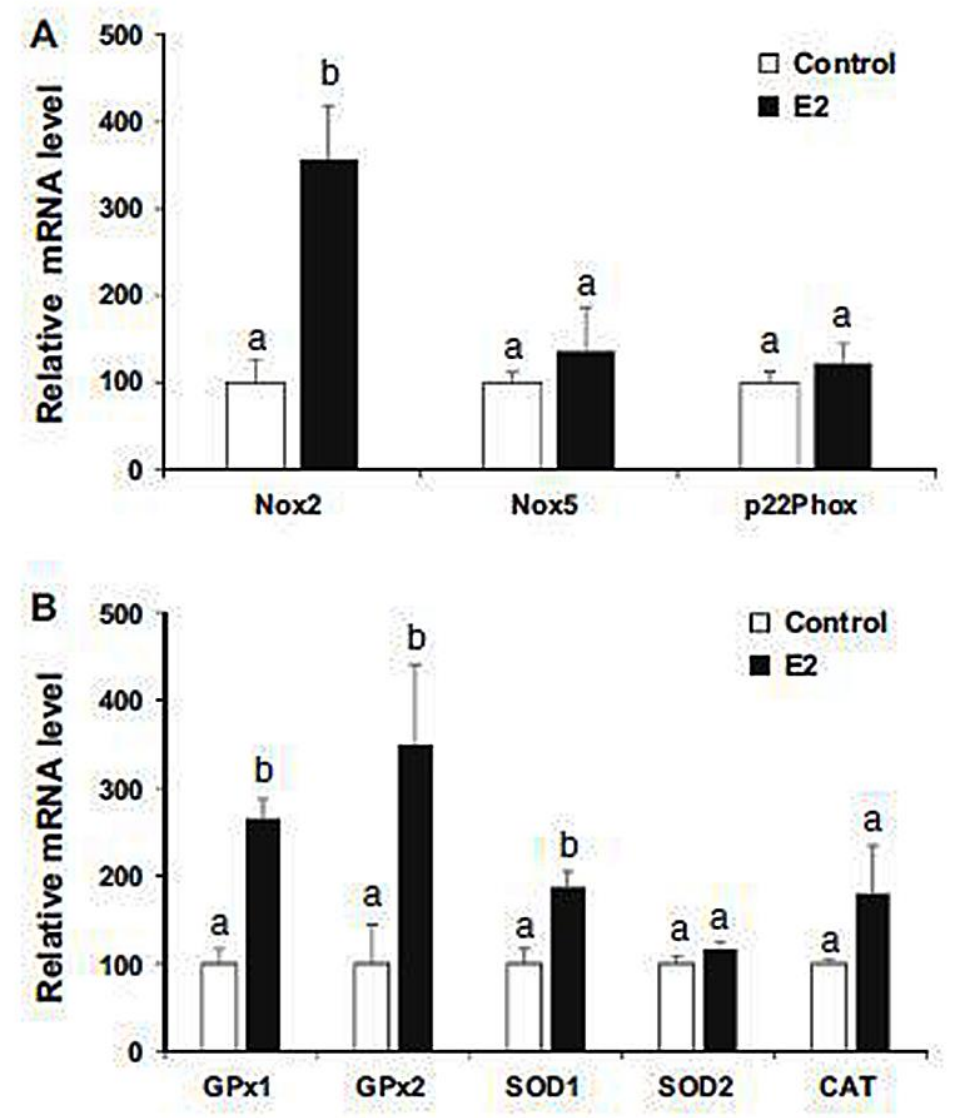

Fig. 1 Pro or antioxidant enzyme and p22phox mRNA expression in short-term E2-treated MCF7L cell line. The stable MCF7L transfectant was obtained by stably transfecting MCF-7 cells with the luciferase gene under the control of a CMV promoter. MCF7L cells were hormone-deprived during 4 days before treatment. They were subsequently placed in DCC medium containing vehicle alone (ethanol) or E2 $10 \mathrm{nM}$ for $24 \mathrm{~h}$. Then, expression of: a prooxidant enzyme mRNA for Nox2, Nox5 and p22phox or b antioxidant enzyme mRNA for GPx1, GPx2, SOD1, SOD2 and CAT were assessed by qPCR analysis as described in "Materials and methods". MCF7L cell line was set at $100 \%$ (mean \pm SEM). Different index letters mean p $\backslash 0.05$ 
The MCF7L cell line was initially designed for subsequent in vivo experiments in nude mice and was generated by stable transfection of MCF-7 cells with a plasmid bearing the luciferase coding sequence under the control of the constitutive CMV promoter. As MCF7L cell line was a clonal one, it was expected to be more homogenous than the parental MCF-7 cell line. The presence of the luciferase gene had no effect on growth properties of the cells (data not shown). In this MCF7L cell line, we found that only Nox2, Nox5 and p22phox were expressed, whereas Nox1 was barely undetectable. As a first step, the hormone sensitivity of these pro or antioxidant enzymes was assessed by analyzing their responsiveness to a shortterm (24 h) estradiol stimulation of MCF7L cells (Fig. 1a, b). Nox2, GPx1, GPx2 and SOD1 mRNA expression levels were increased by estradiol treatment, whereas those for Nox5,

SOD2, CAT and the subunit p22phox mRNA were not significantly affected

\section{Characterization of long-term (LT) treated MCF7L cells}

As described in "Materials and methods", MCF7LOHTLT cell lines were obtained after 3 months of culture in DCC medium with $10 \mathrm{nM} \mathrm{OHT} \mathrm{(Fig.} \mathrm{2).} \mathrm{During} \mathrm{a} \mathrm{1-month} \mathrm{period} \mathrm{after}$ the beginning of OHT treatment, the growth speed of the cells was considerably reduced, and thereafter progressively increased again, indicating that a resistance process occurred.

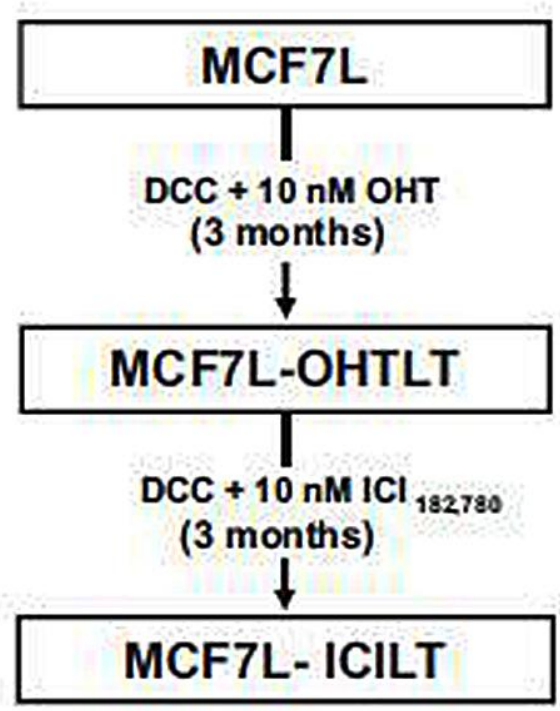

Fig. 2 MCF-7-derived cell lines. MCF7L cells were treated with $10 \mathrm{nM}$ OHT in DCC medium for 3 months and the resulting culture was named MCF7L-OHTLT (LT long-term treated). The MCF7LOHTLT cell line was subsequently treated 3 more months with ICI182,780, the resulting culture being named MCF7L-ICILT cell line

In order to mimic the sequential treatment of patients that would have been treated first with tamoxifen and then with fulvestrant, the MCF7L-ICILT cell line was obtained from MCF7LOHTLT after 3 extra months treatment with $10 \mathrm{nM}$ of ICI182,780. Growth properties of the cell lines were evaluated by using the MTT assay after 7 days of hormone treatment (Fig. 3a). Growth of long-term treated (LT) cells (i.e., OHTLT and ICILT) was no longer stimulated by E2 treatment (Fig. 3a), although all cell lines bear similar levels of hormonally regulated estrogen receptor (Fig. 3b). Growth inhibition obtained with 4-hydroxytamoxifen (OHT) was slightly less in MCF7L-OHTLT cells than in parental MCF7L cells, and did not occur in MCF7- ICILT cells. Interestingly, the growth of MCF7-ICILT cells was no longer inhibited 
by the ICI182,780 compound, contrary to the growth of MCF7-OHTLT that remained inhibited to the same extent than that of MCF7L cell line. In conclusion, while anti-estrogen inhibition profile of MCF7L or OHTLT cells growth was quite similar, that corresponding to MCF7-ICILT was clearly different.

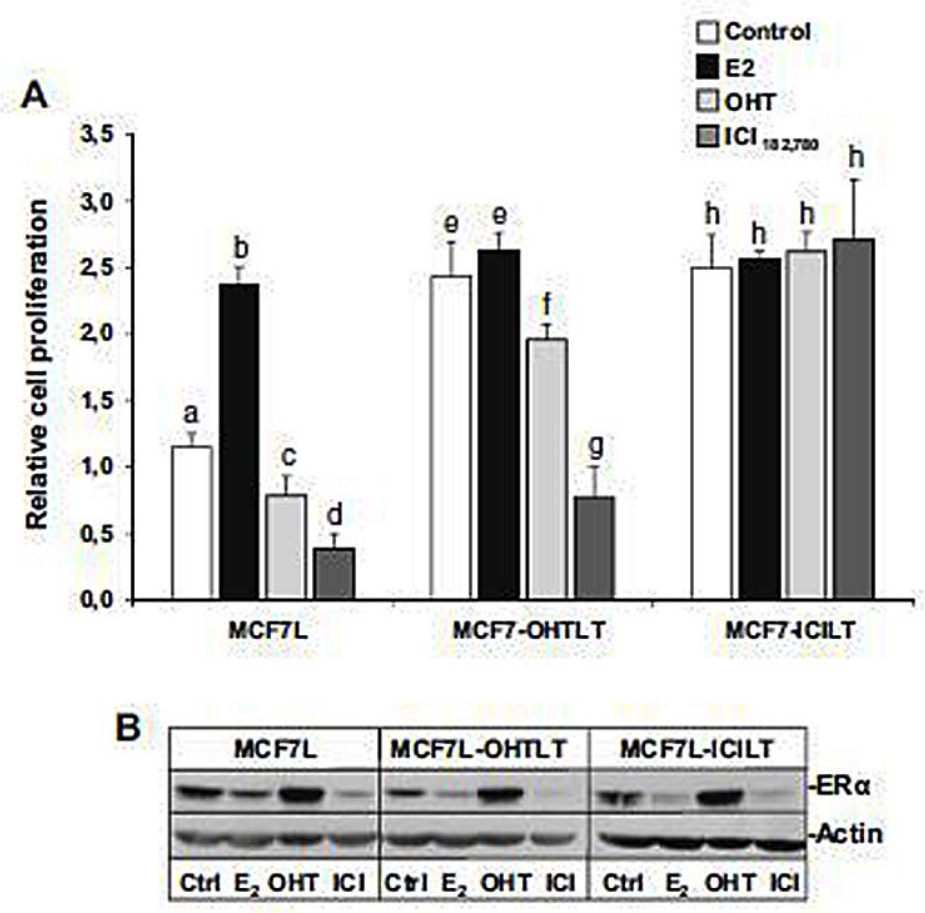

Fig. 3 Growth properties and estrogen receptor alpha expression in MCF-7-derived cells after long-term treatment with anti-estrogens. a Cell proliferation was analyzed (see "Materials and methods"') at day 7 after treatment with the indicated ER ligand or vehicle alone (control) in: MCF7L, MCF7L-OHTLT and MCF7L-ICILT cells (mean \pm SEM). b The different cell lines were treated with the indicated ER ligands or vehicle alone for $24 \mathrm{~h}$ before analysis of Era expression by western blotting as described in "Materials and methods". Different index letters mean p $\backslash 0.05$

\section{Basal ROS production in long-term treated cell lines}

The basal level of ROS was carefully assessed in the different cell lines, by using two different methods. Global intracellular ROS production of living cells was evaluated by measuring the fluorescence of DCFH-DA (Fig. 4a), and global superoxide anion production of solubilized membranes by measuring lucigenin-derived chemiluminescence in the presence of a fixed and saturating concentration of NADPH (Fig. 4b).

Interestingly, both methods gave comparable relative levels of ROS production, indicating that the relative superoxide anion production level (measured by lucigenin method) is representative of the global relative level of ROS when measured by DCFH-DA method. The level of ROS produced by MCF7L-ICILT cell line was significantly lower than that produced by MCF7L parental or MCF7L-OHTLT cell lines. Importantly, for both methods, the extra 3 months of fulvestrant treatment that followed OHT treatment, led to a reduced by half of the amount of ROS. 

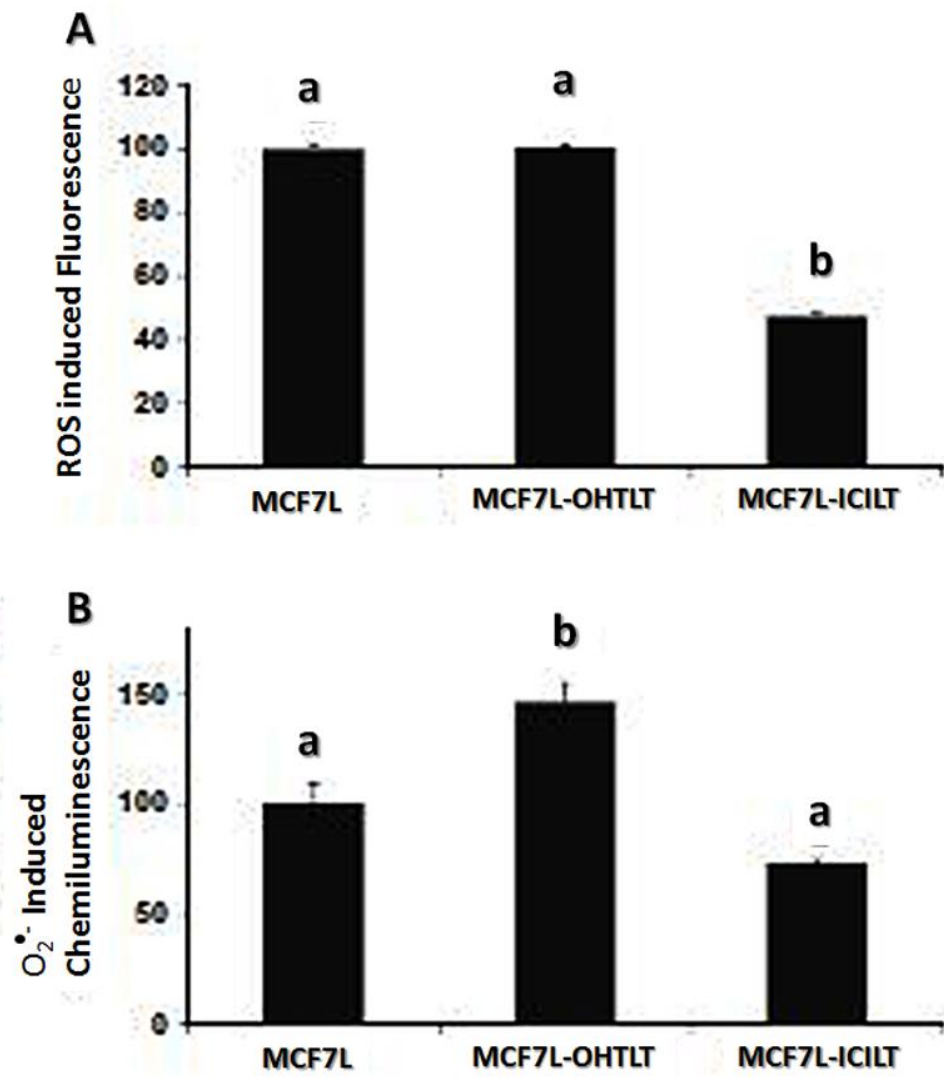

Fig. 4 ROS production in long-term treated cell lines. All cell lines were hormone-deprived during 4 days in DCC medium before being processed for ROS determination. a After hormone deprivation, cells were seeded in 96-well plates, and $24 \mathrm{~h}$ after, basal intracellular ROS determination was assessed by using either DCFH-DA method (mean \pm SEM of 16 independent measurements) or b lucigenin chemiluminescence method (to measure the level of superoxide anion) as described in "Materials and methods". Surfaces under curves (for t $\ 1500 \mathrm{~s}$ ) were estimated and results were expressed as the surface obtained in the presence of DPI minus the one obtained in absence of DPI. MCF7L cell line was set at $100 \%$ (mean \pm SEM). Different index letters mean p 10.05

\section{Pro or antioxidant enzymes and p22phox mRNA expression after a long-term anti- estrogen treatment}

Before mRNA processing, cells were hormone-deprived in DCC medium during 4 days in order to carry out cell analysis in equivalent conditions. Concerning pro-oxidant players, long-term OHT treatment led to a threefold increase level of Nox2 mRNA (MCF7L-OHTLT cells). This increased level was diminished by about $33 \%$ after a subsequent 3-month treatment with the ICI compound (Fig. 5a).

The p22phox accessory protein mRNA level was lowered by about $50 \%$ after OHT long-term treatment, but dropped drastically (10.5\%) after a long-term ICI182,780 treatment (Fig. 5a). Nox 5 mRNA level was not significantly modified by either long-term estrogen deprivation or anti-estrogen treatment.

Concerning antioxidant players (Fig. 5b), in all cell lines analyzed, SOD1 mRNA levels were not significantly different and SOD2 level was slightly lower in MCF7-ICILT. The main 
differences concerned GPx1, GPx2 and CAT mRNA levels. More specifically, when compared to the level in MCF7L cells, GPx2 mRNA level was higher in MCF7L-OHTLT and decreased in MCF7L-ICILT cell line. It appears that on the whole, mRNA expression in longterm fulvestrant treated cells was lower than in OHT treated or in untreated MCF7L cells.


Fig. 5 Pro or antioxidant enzyme and p22phox mRNA expression in long-term treated cell lines. All long-term (LT) cell lines were hormone-deprived during 4 days in DCC medium before being processed for qPCR mRNA expression analysis of: a pro-oxidant enzymes: Nox2, Nox5, and the subunit p22phox or b antioxidant enzymes: GPx1, GPx2, SOD1, SOD2, and CAT. MCF7L cell line was set at $100 \%$ (mean \pm SEM). Different index letters mean p $\backslash 0.05$

\section{Nrf2 cofactor and Keap1 inhibitor mRNA expression in long-term treated cells}

Nrf2 and its inhibitory partner Keap1 are central players of antioxidant enzymes gene expression. The mRNA level of Keap1 and Nrf2 was practically identical in all MCF7Lderived cell lines (Fig. 6). However, the regulation of Nrf2- Keap1 activity is complex and not solely dependent on the level of their mRNA or protein expression. We thus also investigated the level of expression of NQO1 gene, which is a direct target for the cofactor Nrf2 (Fig. 6). We found an intermediate level of NQO1 mRNA in anti-estrogen treated cell lines. This tendency seems consistent with the global level of antioxidant enzymes mRNA that was observed in long-term anti-estrogen treated cell lines. 


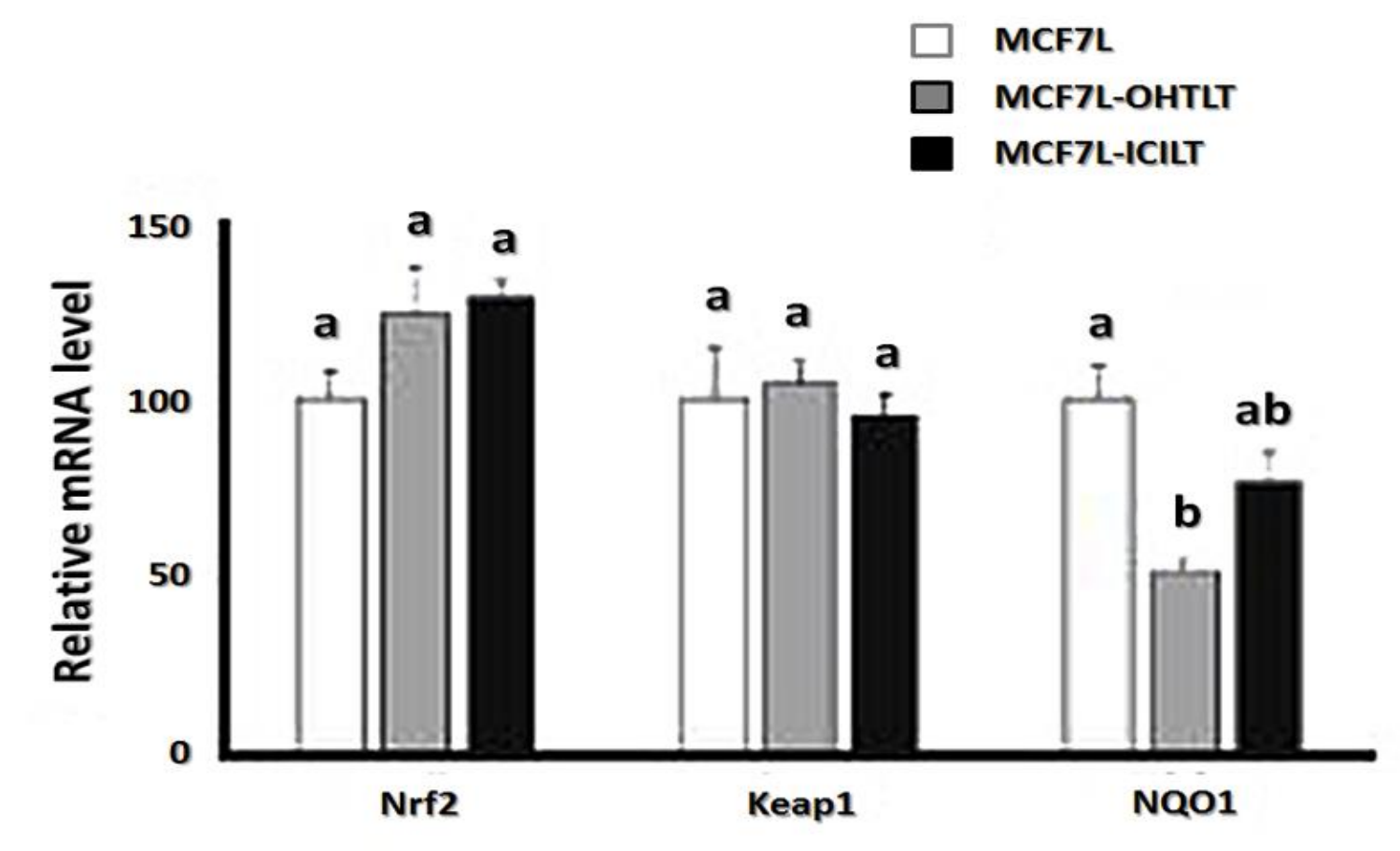

Fig. 6 Nrf2, Keap1 and NQO1 mRNA expression levels in long-term treated cell lines. Long term cell lines were hormone-deprived during 4 days in DCC medium and then expression levels of Nrf2, Keap1 or NQO1 mRNA were assessed by qPCR analysis as described in "Materials and methods". MCF7L cell line was set at $100 \%$ (mean \pm SEM). Different index letters mean p 10.05

\section{Effect of antiradical tempol treatment}

When MCF7L-OHTLT were treated for 3 months with ICI182,780 (MCF7L-ICILT), we observed the disappearance of ICI182,780 growth inhibitory action (Fig. 3) associated to a decrease in ROS levels (Fig. 4). In order to reinforce the link between these two events, MCF7L-OHTLT and MCF7L-ICILT cells were treated or not with the antiradical agent tempol (at 0.25 or $0.5 \mathrm{mM}$ ) in the presence or absence of ICI182,780 during 5 days (Fig. 7). The measurement of intracellular ROS by DCFH-DA assay showed that after $24 \mathrm{~h}$ of $0.5 \mathrm{mM}$ tempol, the ROS content of MCF7-OHTLT cells decreased by $38 \%$ (data not shown). As shown in Fig. 7a, tempol exerted an inhibitory effect on the growth of the two cell lines as already described in MCF-7 cells [27]. Interestingly, it appeared that the antiproliferative effect of tempol (at the intermediate dose $0.25 \mathrm{mM}$ ) was more obvious in MCF7L-ICILT than in MCF7L-OHTLT cells. This could be related to the decrease in ROS levels observed in MCF7L-ICILT (Fig. 4). Indeed, one can hypothesize that a low level of ROS allows a better efficiency of the antiradical activity of tempol and, as a consequence, leads to an increase of its antiproliferative effect. Thus, this first observation could support a role of ROS level in the proliferative properties of MCF7L-ICILT cells. Such a role was further supported by data shown in Fig. 7b, which revealed that, in MCF7LOHTLT, tempol treatment decreased the antiproliferative activity of ICI treatment. Altogether, these data support the idea that ICI resistance could be linked to a decrease in ROS levels. 

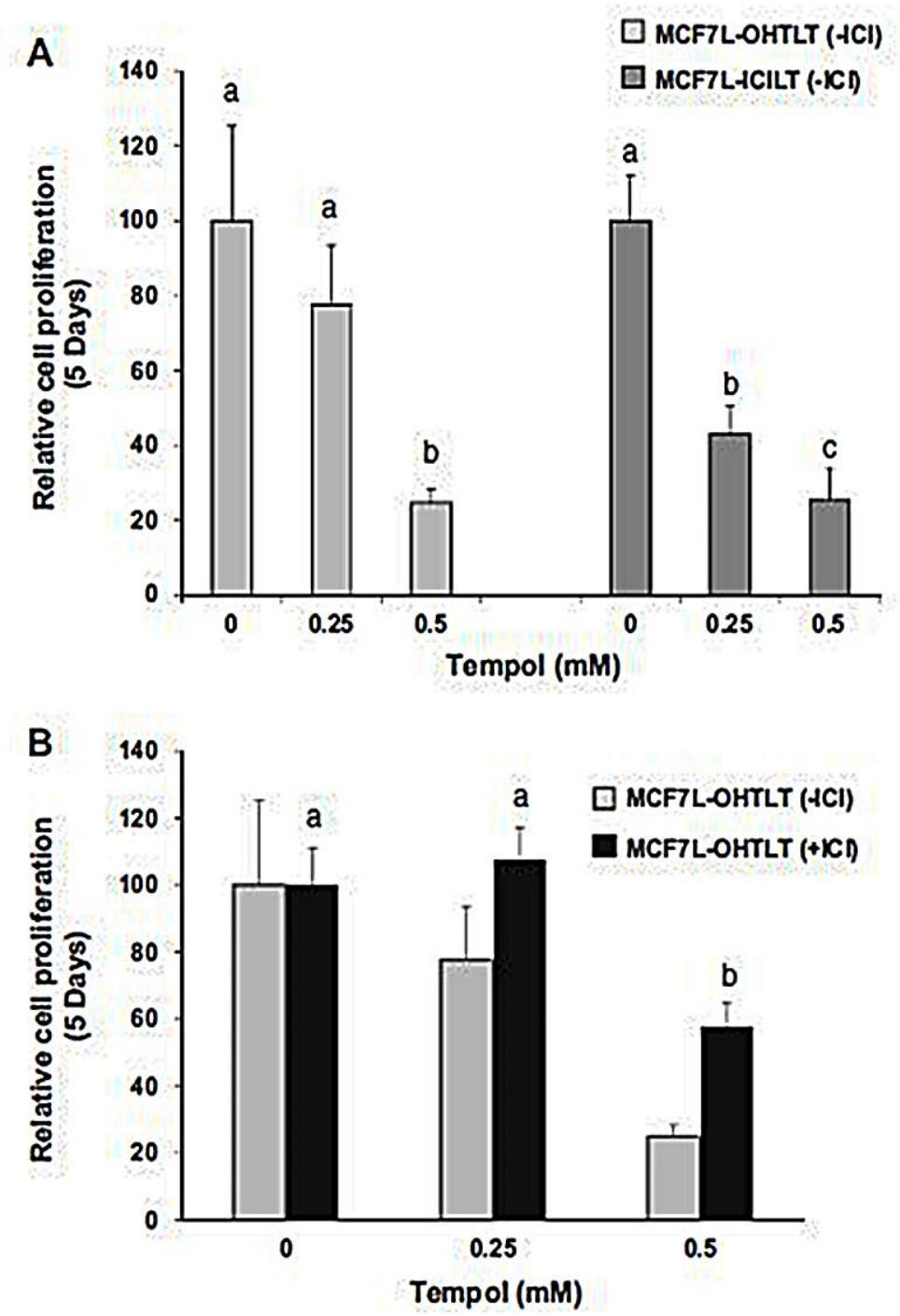

Fig. 7 Effect of antiradical tempol treatment in MCF7L-OHTLT or MCF7L-ICILT cells. Cells were hormone-deprived during 4 days in DCC medium. They were then seeded $(10,000$ cells/well) in 96-well plates and treated during 5 days with the indicated tempol concentrations, in the presence or absence of ICI182,780 compound. Cell density at day 6 was assessed by MTT as described in "Materials and methods". Results were normalized to the cell density at day 0. a Relative proliferation of MCF7L-OHTLT and MCF7L-ICILT cell lines treated with increasing concentrations of tempol in the absence of ICI182,780. b Effect of tempol on the response of MCF7L_OHTLT cells to ICI182,780 treatment. In a and b the control (0 tempol) was set at $100 \%$ (mean \pm SEM). Different index letters mean p $\backslash 0.05$

\section{Discussion}

In this study, we investigated the pro or antioxidant status of a breast cancer MCF-7 derived cell line (MCF7L) after long-term anti-estrogen treatments. As a first step, it was shown that mRNA expression of several enzymes involved in the redox status or the cell (e.g., Nox2, GPx1, GPx2, SOD1, CAT) were induced by $24 \mathrm{~h}$ of estradiol treatment. The hormone sensitivity of such players suggests that steroid hormones may play a critical role in the redox equilibrium of the cell and the subsequent level of ROS. These results were in accordance 
with those of Mobley who found that SOD and GPx enzyme activities were raised after estradiol treatment in MCF7 cells [11]. However, there was a discrepancy concerning the CAT enzyme whose activity was found to decrease after E2 treatment, perhaps due to extra transcriptional effects. Such hormonal effect of estradiol on SOD enzymes was also observed in vascular smooth muscle cells and was expected to be involved in the vasoprotective effect of this hormone in females [28]. Moreover, short-term effects of estradiol appear to be tissue dependent, because in human endothelial cells, estradiol led to an inhibition of NADPH oxidase expression [29]. Short-term effects of both OHT and fulvestrant on Nox2 and p22phox mRNA expression were also tested, but no significant variations were observed after $24 \mathrm{~h}$ of treatment (data not shown).

In both, short-term and long-term treatments, significant changes of Nox 2 mRNA level were observed, whereas that for Nox5 remained unchanged, indicating a hormone sensitivity of the former. However, Nox 2 mRNA was raised either by short-term estradiol treatment or by longterm estradiol deprivation (data not shown) as well as long-term anti-estrogen treatment (also performed in estradiol stripped serum), the highest level being attained after long-term OHT treatment. Such transcriptional effects might reflect a cellular stress that is induced for each of these three conditions.

The prominent effect induced by long-term anti-estrogen treatment was the sharp decrease of p22phox accessory protein mRNA that appeared after 3 months of fulvestrant treatment, whereas short-term treatments did not show any significant transcriptional hormone sensitivity for this mRNA. As only partial $(50 \%)$ inhibition was observed after OHT longterm treatment, complete inhibition of p22phox mRNA transcription appears to be specifically induced by the subsequent fulvestrant treatment. Beside the well-known implication of p22phox in NADPH oxidase activity, its involvement in renal or pancreatic cancers has also been described in the literature [30]. In the case of renal cancer, a p22phoxdependent inactivation of the tumor suppressor gene tuberin was evidenced, indicating that this protein might be associated with a cancerous phenotype [30]. In the case of pancreatic cancer, up regulation of $\mathrm{p} 22$ phox by growth factors led to a pro-survival mechanism with an increase of NADPH-oxidase activities and inhibition of apoptosis [31]. In the light of these two examples, one can hypothesize that inhibition of p22phox expression induced by longterm fulvestrant treatment might hinder the proliferative capacity of breast cancer cells.

Concerning the implication of p22phox inhibition on ROS generation, we noticed that three extra months of fulvestrant treatment led to a marked and significant decrease of ROS level in MCF7L-ICILT cell line when compared to MCF7L-OHTLT.

In spite of these investigations, it remains difficult to pull things together into a coherent whole. Firstly, there are several possible sources of cellular ROS other than those produced by NADPH oxidases, in particular mitochondrial one. Secondly, NADPH oxidases are not all dependent on p22phox subunit. Nevertheless, when mRNA expression of five antioxidant enzymes are compared, on the whole, we found that mRNA expression in MCF7L-ICILT is either lower or not significantly different than the corresponding mRNA expression in MCF7OHTLT. Mention may be made of the very low level of GPx 2 in MCF7L-ICILT compared to the high level in MCF7-OHTLT. One can hypothesize that the level of antioxidant enzymes has gradually adapted to the lower level of ROS reached after the long-term fulvestrant treatment. 
Study of Cho [18] suggests the possibility of a causal link between ROS level and antihormone resistance. In our study, we used tempol, which appeared to be the more convenient antiradical compound. Indeed, growth analysis by using chemical ROS inhibitors like DPI or apocynin is often hampered by their cytotoxicity or their adverse effects [32, 33]. However, whereas interpretation of our results was complicated by the antiproliferative effect of tempol on cells, they support a possible link between ROS and ICI182,780 sensitivity. To go further in such a question, the use of cell lines with stable and inducible expression of antioxidant enzymes such as SOD might be a way to bypass the antiproliferative effect of tempol and allow an easier analysis of the relationships between ROS level, apoptosis and growth control in long-term treated breast cancer cell lines. In addition, we noticed a difference of growth sensitivity between MCF7L-OHTLT and MCF7L-ICILT cell lines, when treated with $0.25 \mathrm{mM}$ tempol. Regardless of its mechanistic significance, such treatment with this intermediate concentration of tempol might be a way to obtain a noticeable growth inhibition of cells that are proven to be resistant to both OHT and ICI182,780. Such effect could have a potential therapeutic value. This first study needs to be further investigated in order to strengthen our comprehension of the relationships between ROS production and ICI182,780 sensitivity in breast cancer cells. First, the analysis of several independent resistant clones issued from the same cell line would give a statistical foundation to our data. Moreover, subsequent analysis of other long-term anti-estrogen treated breast cancer cell lines, as well as anti-estrogen-resistant tumors in animals would tell us if inhibition of both p22phox and ROS is a general phenomenon induced by long-term ICI182,780 treatment.

In addition, as the present study mainly focused on the transcriptional effects of hormone treatments, additional mechanistic aspects at the level of protein regulation (including posttranslational modifications, protein stability, enzyme activity) need also to be addressed in a future study.

In conclusion, long-term anti-estrogen treatments were associated with important changes in the levels of pro or antioxidant enzymes expression and ROS concentration. Interestingly, when compared to OHT treatment alone, a drastic inhibition of p22phox mRNA was observed after long-term fulvestrant extra treatment that was accompanied by a diminution of the ROS level. Both effects may be beneficial in terms of treatment in patients treated with fulvestrant.

\section{Compliance with ethical standards}

Conflict of interest : The authors declare that they have no conflict of interest.

\section{References}

1. Storz P. Reactive oxygen species in tumor progression. Front Biosci. 2005;10:1881-96.

2. Waris G, Ahsan H. Reactive oxygen species: role in the development of cancer and various chronic conditions. J Carcinog. 2006;5:14.

3. Block K, Gorin Y. Aiding and abetting roles of NOX oxidases in cellular transformation. Nat Rev Cancer. 2012;12:627-37.

4. Weyemi U, Redon CE, Parekh PR, Dupuy C, Bonner WM. NADPH oxidases NOXs and DUPXs as putative targets for cancer therapy. Anticancer Agents Med Chem. 2013;13:50214.

5. Brieger K, Schiavone S, Miller FJ Jr, Krause KH. Reactive oxygen species: from health to disease. Swiss Med Wkly. 2012;142:w13659.

6. Benhar M, Engelberg D, Levitzki A. ROS, stress-activated kinases and stress signaling in cancer. EMBO Rep. 2002;3:420-5. 
7. Tochhawng L, Deng S, Pervaiz S, Yap CT. Redox regulation of cancer cell migration and invasion. Mitochondrion. 2013;13: 246-53.

8. Thannickal VJ, Fanburg BL. Reactive oxygen species in cell signaling. Am J Physiol Lung Cell Mol Physiol. 2000;279:L1005-28.

9. Kimura H, Sawada T, Oshima S, Kozawa K, Ishioka T, Kato M. Toxicity and roles of reactive oxygen species. Curr Drug Targets Inflamm Allergy. 2005;4:489-95.

10. Bedard K, Krause KH. The NOX family of ROS-generating NADPH oxidases:

physiology and pathophysiology. Physiol Rev. 2007;87:245-313.

11. Mobley JA, Brueggemeier RW. Estrogen receptor-mediated regulation of oxidative stress and DNA damage in breast cancer. Carcinogenesis. 2004;25:3-9.

12. Jain S, Saxena D, Kumar PG, Koide SS, Laloraya M. Effect of estradiol and selected antiestrogens on pro- and antioxidant pathways in mammalian uterus. Contraception.

1999;60:111-8.

13. Borras C, Gambini J, Gomez-Cabrera MC, Sastre J, Pallardo FV, Mann GE, et al. 17betaoestradiol up-regulates longevity-related, antioxidant enzyme expression via the ERK1 and ERK2[MAPK]/ NFkappaB cascade. Aging Cell. 2005;4:113-8.

14. Razandi M, Pedram A, Jordan VC, Fuqua S, Levin ER. Tamoxifen regulates cell fate through mitochondrial estrogen receptor beta in breast cancer. Oncogene. 2013;32:3274-85.

15. Ponzone R, Biglia N, Jacomuzzi ME, Mariani L, Dominguez A, Sismondi P. Antihormones in prevention and treatment of breast cancer. Ann N Y Acad Sci. 2006;1089:143-58.

16. Badia E, Oliva J, Balaguer P, Cavailles V. Tamoxifen resistance and epigenetic modifications in breast cancer cell lines. Curr Med Chem. 2007;14:3035-45.

17. Clarke R, Leonessa F, Welch JN, Skaar TC. Cellular and molecular pharmacology of antiestrogen action and resistance. Pharmacol Rev. 2001;53:25-71.

18. Cho SK, Pedram A, Levin ER, Kwon YJ. Acid-degradable coreshell nanoparticles for reversed tamoxifen-resistance in breast cancer by silencing manganese superoxide dismutase (MnSOD). Biomaterials. 2013;34:10228-37.

19. Badia E, Docquier A, Busson M, Lapierre M, Pujol P, Balaguer P, et al. Long-term treatment with the pure anti-estrogen fulvestrant durably remodels estrogen signaling in BG-1 ovarian cancer cells. J Steroid Biochem Mol Biol. 2012;132:176-85. Breast Cancer (2016) 23:692-700 .

20. Johnston SJ, Cheung KL. Fulvestrant - a novel endocrine therapy for breast cancer. Curr Med Chem. 2010;17:902-14.

21. Croxtall JD, McKeage K. Fulvestrant: a review of its use in the management of hormone receptor-positive metastatic breast cancer in postmenopausal women. Drugs. 2011;71:363-80. 22. Trachootham D, Lu W, Ogasawara MA, Nilsa RD, Huang P. Redox regulation of cell survival. Antioxid Redox Signal. 2008;10:1343-74.

23. Badia E, Duchesne MJ, Fournier-Bidoz S, Simar-Blanchet AE, Terouanne B, Nicolas JC, et al. Hydroxytamoxifen induces a rapid and irreversible inactivation of an estrogenic response in an MCF-7-derived cell line. Cancer Res. 1994;54:5860-6.

24. Oliva J, El Messaoudi S, Pellestor F, Fuentes M, Georget V, Balaguer P, et al. Involvement of HP1alpha protein in irreversible transcriptional inactivation by antiestrogens in breast cancer cells. FEBS Lett. 2005;579:4278-86.

25. Rossary A, Arab K, Goudable J, Steghens JP. Re'gulation de l'activite' des NADPH oxydases en pre'sence d'acides gras. Ann Biol Clin. 2007;65:33-40.

26. Juhasz A, Ge Y, Markel S, Chiu A, Matsumoto L, van Balgooy J, et al. Expression of NADPH oxidase homologues and accessory genes in human cancer cell lines, tumours and adjacent normal tissues. Free Radic Res. 2009;43:523-32. 
27. Gariboldi MB, Terni F, Ravizza R, Meschini S, Marra M, Condello M, et al. The nitroxide Tempol modulates anthracycline resistance in breast cancer cells. Free Radic Biol Med. 2006;40: 1409-18.

28. Wassmann K, Wassmann S, Nickenig G. Progesterone antagonizes the vasoprotective effect of estrogen on antioxidant enzyme expression and function. Circ Res. 2005;97:104654.

29. Wagner AH, Schroeter MR, Hecker M. 17beta-estradiol inhibition of NADPH oxidase expression in human endothelial cells. Faseb J. 2001;15:2121-30.

30. Block K, Gorin Y, New DD, Eid A, Chelmicki T, Reed A, et al. The NADPHoxidase subunit p22phox inhibits the function of the tumor suppressor protein tuberin. Am J Pathol. 2010;176:2447-55.

31. Edderkaoui M, Nitsche C, Zheng L, Pandol SJ, Gukovsky I, Gukovskaya AS. NADPH oxidase activation in pancreatic cancer cells is mediated through Akt-dependent up-regulation of p22phox. J Biol Chem. 2011;286:7779-87.

32. Park SE, Song JD, Kim KM, Park YM, Kim ND, Yoo YH, et al. Diphenyleneiodonium induces ROS-independent p53 expression and apoptosis in human RPE cells. FEBS Lett. 2007;581:180-6.

33. Riganti C, Costamagna C, Bosia A, Ghigo D. The NADPH oxidase inhibitor apocynin (acetovanillone) induces oxidative stress. Toxicol Appl Pharmacol. 2006;212:179-87. 700 Breast Cancer (2016) 23:692-700 